UMR 5824

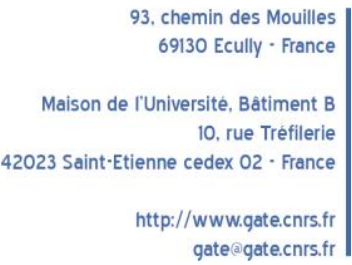

WP 1730 - October 2017

\title{
Regulation and altruism
}

\author{
Izabela Jelovac, Samuel Kembou Nzale
}

\begin{abstract}
:
We study optimal contracts in a regulator-agent setting with joint production, altruistic and selfish agents, and uneasy outcome measurement. Such a setting represents sectors of activities such as education and health care provision. The agents and the regulator jointly produce an outcome for which they all care to some extent that is varying from agent to agent. Some agents, the altruistic ones, care more than the regulator does while others, the selfish agents, care less. Moral hazard is present due to the agent's effort that is not contractible. Adverse selection is present too since the regulator cannot a priori distinguish between altruistic and selfish agents. Contracts consist of a simple transfer from the regulator to the agents together with the regulator's input in the joint production. We show that a screening contract is not optimal when we face both moral hazard and adverse selection.
\end{abstract}

\section{Keywords:}

altruism, moral hazard, adverse selection, regulator-agent joint production

\section{JEL codes:}

D64, D86

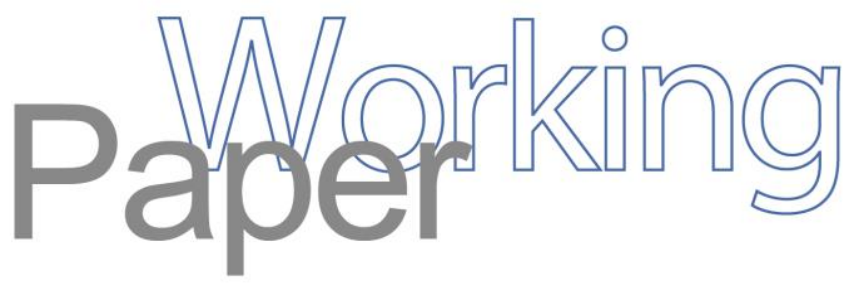




\title{
Regulation and altruism *
}

\author{
Izabela JELOVAC † Samuel KEMBOU NZALE ‡
}

\author{
This version: October 2017
}

\begin{abstract}
We study optimal contracts in a regulator-agent setting with joint production, altruistic and selfish agents, and uneasy outcome measurement. Such a setting represents sectors of activities such as education and health care provision. The agents and the regulator jointly produce an outcome for which they all care to some extent that is varying from agent to agent. Some agents, the altruistic ones, care more than the regulator does while others, the selfish agents, care less. Moral hazard is present due to the agent's effort that is not contractible. Adverse selection is present too since the regulator cannot a priori distinguish between altruistic and selfish agents. Contracts consist of a simple transfer from the regulator to the agents together with the regulator's input in the joint production. We show that a screening contract is not optimal when we face both moral hazard and adverse selection.
\end{abstract}

Keywords: altruism, moral hazard, adverse selection, regulator-agent joint production.

JEL Classification: D64, D86.

*This paper has greatly benefited from comments by Francesca Barigozzi, Paul Belleflamme, Petyo Bonev, David Bardey, Chiara Canta, Rose Anne Devlin, Jocelyn Donze, Mathias Kifmann, Michael Kuhn, Pau Olivella, David Perez Castrillo, Bruno Ventelou, Gijsbert Zwart and seminar participants at the 2016 Meeting of the Association for Public Economic Theory, 15th Annual Canadian Health Economists' Study Group, 17th European Health Economics Workshop, 13th edition of the Augustin Cournot Doctoral Days, 21st Spring Meeting of Young Economists, 2nd meeting of the Austrian Health Economics Association, 37th Journees des Economistes de la Sante Francais, 14th Journees Maurice Marchand des Doctorants en Economie de la Sante. The usual disclaimers apply.

${ }^{\dagger}$ Univ Lyon, CNRS, GATE UMR 5824, Ecully, F-69130, France. izabela.jelovac@cnrs.fr

${ }_{\ddagger}^{\ddagger}$ Aix-Marseille Univ.(Aix-Marseille School of Economics), CNRS, EHESS and Centrale Marseille, F-13002. samuel.kembou-nzale@univ-amu.fr 


\section{Introduction}

In sectors such as education and health care, the measurement of results is uneasy and thus results hardly influence payments or rewards. Moreover, the outcomes in such sectors often depend on the contributions of both the agent and the regulator. The agent can be a teacher or a health care provider and she contributes with her professional effort. The regulator represents the collectivity and he provides inputs such as computers, classrooms, universities, hospitals, etc. Also, the agent and the regulator happen to share the same objectives, at least partially. A teacher cares for the quality of the education and a physician cares for the quality of health care. The agent and the regulator care for these outcomes to some extent, which is varying from one agent to another. If an agent cares more than the regulator does, we call her altruistic. Instead, if she cares less than the regulator does, we call her selfish. This heterogeneity in agent's altruism is in line with the empirical evidence reported by Watt et al. (2014) for teachers and by Brosig-Koch et al. (2017) for physicians. In our setting, the concepts of altruism, mission oriented behavior, professional ethics or public service motivation are actually equivalent.

In this paper, we analyze optimal contracts in a principal-agent setting that reflects the characteristics of such sectors. The principal (he) is a regulator who cares for some outcome jointly produced with the agent (she). The agent's effort and the outcome are not contractible. Moreover, the agent has private information on her altruism level, that is, on the extent to which she shares the regulator's concern for the jointly produced outcome. Therefore, contracts consist of a transfer from the regulator to the agent and of a regulator's input in the joint production. To sum up, we face a problem of moral hazard together with adverse selection.

Our main result indicates that information about altruism is never completely extracted. We compute the optimal contract in three regimes. We refer to first-, secondand third-best contracts, respectively, without any agency problem, with moral hazard only and contracts with moral hazard cum adverse selection. We proceed in this way because it enables to study progressively how the information asymmetry impacts on the regulator-agent relationship. With the first-best contract, regulator's input, effort and transfer do not vary with the altruism level and they are decreasing in the shadow cost of public funds. Altruism does not affect the first-best solution because the latter satisfies a binding agent's budget constraint, which does not include her altruistic benefits. As long as the solution satisfies the budget constraint, it also satisfies the participation constraint, which adds the altruistic component to the budget. The first-best contract would still be optimal if the only agency problem was adverse selection. With the second-best contract, we show that, effort and transfer increase with altruism and the regulator's input is invariant to changes in altruism. In the third-best analysis, we find that no screening contract dominates the second-best contract designed to deal with moral hazard of an 
altruistic agent. Screening according to altruism is not optimal because its only effect would be to increase the informational rent of a selfish agent.

The agent receives the same transfer level and she works with the same level of the regulator's input, no matter whether she is altruistic or selfish. In fact, the monotonicity condition imposes the regulator's input for an altruistic agent to be not lower than that for a selfish agent. Comparative statics on the informational rent of a selfish agent show that, this rent increases (decreases) with the regulator's input for an altruistic agent (selfish agent). This tension forces the regulator to give the same input level to all the types. This result is referred to as "non-responsiveness" because of this tension between optimality and incentives.

To study the impact of moral hazard, we compare the first-best solution with the second-best one. When we move from the first- to the second-best solution, the regulator's input is not distorted. However, the second-best effort and transfer are distorted upward (downward) when altruism and shadow cost of public funds are sufficiently high (low). Note that in the second-best analysis, the representative agent does not internalize the shadow cost of public funds when she decides on effort. Moreover, the marginal benefit she derives from effort increases with her degree of altruism.

We compare first-, second- and third-best solutions to study the impact of adverse selection and moral hazard. Because the third-best contract is a non-separating one, the combination of moral hazard and adverse selection has no impact on agent's effort and on regulator's input. Both third-best effort and input are identical to second-best effort and input. Only the transfer is different. In the second-best case, the transfer compensates each type of agent for her cost of effort. An altruistic agent therefore earns a higher transfer than a selfish agent only because her effort is higher. In the third-best contract, the transfer is unique because the regulator cannot tell the agent's types apart and thus the transfer is the one that compensates the cost of the highest effort, which corresponds to the highest level of altruism. A selfish agent thus earns an informational rent.

An important driver of our results is our modelling of the agent's participation and budget constraints. The difference between the participation constraint and the budget constraint is the altruistic part of the agent's utility. A classical participation constraint grants the agent a utility that is not lower than a reservation utility, which we normalize to zero. This classical participation constraint includes the altruistic component of the agent's utility. Such a participation constraint appears in the analysis by Jack (2005). It implicitely assumes that the altruistic satisfaction of contributing to the outcome can only be obtained within the principal-agent relationship. It also implicitely assumes that an altruistic agent is ready to pay to enter the principal-agent relationship. We rather opt for a stronger version of the participation constraint. To avoid any confusion, we call it a budget constraint and it grants the agent a payment that is not lower than her 
personnal cost of effort. It is equivalent to the minimum profit constraint in Choné \& Ma (2011). The only difference with a classical budget constraint is that it does not include the altruistic part of the utility. By construction, it implies the classical participation constraint. We can interpret this budget constraint as a legal constraint according to the idea that work must pay. We can also interpret it as a participation constraint if we consider that the altruistic part of the utility enters not only the agent's utility withing the principal-agent relationship, but also her reservation utility. This approach is valid if the agent can contribute to the outcome outside of the principal-agent relationship. In this case, individual rationality would make her volunteer rather than pay for working.

The rest of the paper is organized as follows. In the following subsection, we describe the related literature. In section 2, we set up the model. In Section 3, we analyze optimal contracts when there is no asymmetry of information. In section 4, we analyze optimal contracts with a focus on moral hazard: the agents' effort is not contractible. In section 5 , we derive optimal contracts with a focus on both moral hazard and adverse selection: the agent has private information on her altruism and his effort is not contractible. We conclude in section 6. Proofs together with an illustrating example are in Appendix.

\subsection{Related literature}

The approach our paper uses to capture the issue of regulation and altruism is to consider that the regulator and the agent participate in a joint production with private information on the agent's altruism and non-observability of effort. In this literature review, we discuss how different is our model compared to classical principal-agent models used in the literature. We also discuss our result on the non-optimality of screening in regards to other papers in the principal-agent literature that explain why screening can sometimes be non advantageous.

The first strand is the literature on joint production (Dor \& Watson (1995); Jelovac \& Macho-Stadler (2002)). The closest paper is that of Jelovac \& Macho-Stadler (2002). They consider a model in which two agents, a hospital and a physician, both participate in the production of health services. The agency problem the paper deals with is twosided moral hazard. There is moral hazard on the physician's effort and on the hospital's investment. The aim of the paper is to analyze and compare the performance of two possible organizations for health services: a centralized structure in which the regulator contracts with both agents (hospital and physician) and a decentralized structure in which he contracts with the hospital only, delegating to this latter the authority to contract with physicians. Unlike Jelovac and Macho-Stadler however, we account for altruism by integrating it in the objective of the agent. While their paper deals with two sided moral hazard on the two inputs of the joint production function, our model rather considers moral hazard on one input and adverse selection on the altruism parameter that enters 
the agent's objective function.

The second strand of the literature to which our paper relates is the literature on adverse selection on altruism. Many papers in the literature consider moral hazard with altruism. However, heterogeneity in altruism is relatively new and scarce in the literature (see Macho-Stadler \& Pérez Castrillo (2016)'s discussions on extensions of moral hazard models and Köszegi (2014)'s review on behavioral contract theory). Experimental studies report that agents differ in their levels of altruism. For example, Brosig-Koch et al. (2017) report heterogeneity in altruism among medical and non-medical subjects. Some other theoretical works integrate the altruistic component in their analysis without explicitly considering its heterogeneity in the population (see Biglaiser \& Ma (2007) and Naegelen \& Mougeot (2011) among others).

Theoretical papers to which our study directly relates are Jack (2005), Choné \& Ma (2011), Liu \& Ma (2013) and Barigozzi \& Burani (2016). Jack (2005) studies optimal contracts under both adverse selection on altruism and moral hazard on agent's effort. He studies how the regulator can use the cost-sharing parameter and the transfer to deal with these two informational problems. Choné \& Ma (2011) consider a physicianconsumer relationship with heterogeneity on both altruism and patient's benefit. They show that, without heterogeneity on consumer's benefit, the regulator can impose the first-best quantity even under unknown altruism. Our result on non-optimality of a screening contract corroborates Chon and Ma's results if their setting accounted only for unknown altruism. In Liu \& Ma (2013)'s setting, the physician-consumer relationship also takes into account commitment, risk aversion and insurance. They show that the first best (same transfer regardless of the provider's altruism) can be implemented in a "commitment game" where the physician commits to treatment decisions at contracting. Screening appears only in the non-commitment game where more altruistic providers earn positive profits. Barigozzi \& Burani (2016) analyze optimal contracts between a hospital and health professionals privately informed about their ability and altruism. They introduce difference in ownership structures as well as mission of hospitals. The first big difference between our paper and these four papers is that we have a joint production framework. In our framework, the regulator and the agent both contribute to the non-contractible outcome. The second difference is the fact that we do not assume that the variable under the agent's control is contractible. Assuming that the regulator can neither contract on agent's effort nor on the produced outcome makes our analysis different from what has been done in earlier work in the literature using principal-agent frameworks. Therefore, our results really complement the results of all these papers with this alternative setting that recognizes that outcome cannot be accurately measured and production is joined.

The third strand of the literature to which our paper is related analyzes why screening 
can be sub-optimal. A first answer is given by Salant (1989). He studies the circumstances under which it is not optimal for a monopolist to use second-degree price discrimination, that is, screening between consumers on the basis of price and quality. This paper reconciles the striking result of Stokey (1979) with that of Spence $(1977,1980)$ and Mussa \& Rosen (1978). Stokey (1979) shows in an inter-temporal model, that it is never advantageous to induce self selection even though it is feasible. Spence $(1977,1980)$ and Mussa \& Rosen (1978) show on the contrary that the unique optimal strategy for a monopolist is to induce self selection among heterogeneous consumers. Salant (1989) identifies conditions, which are necessary for price discrimination to occur when the optimization problem is weakly concave. This reference is useful in the sense that it helps make reasonable modelling assumptions in adverse selection models so that the possible result on the disadvantage of screening is not an artefact of the model. Our paper does not fall in the cases studied in Salant (1989). In fact, the author considers adverse selection on one parameter : either quantity to reflect Spence's model, or quality to reflect Musa-Rosen's model, or date at which the good is purchased to reflect Stokey's model. The paper also considers price as being the instrument to solve adverse selection. In our analysis, we do have adverse selection on one parameter: the agent's altruism. However, we have two instruments to solve adverse selection: transfer and regulator's input. Moreover, as already mentioned, we have neither the outcome nor the effort being contractible variables.

The second reason for the non-optimality of screening is provided in Guesnerie \& Laffont (1984)'s general analysis of principal-agent models with a continuum of types. They define non-responsiveness as situations in which there exists a strong conflict between the principal's desire to have the more efficient agent produce more than the inefficient one for pure efficiency reasons and the monotonicity condition imposed by asymmetric information (see Laffont \& Martimort (2009)). This conflict is identified as being the cause of the non-optimality of a screening contract in our setting. As already mentioned, monotonicity implies that the regulator should provide altruistic agents with a higher input while optimality requires input for less altruistic providers to be be higher. This forces the regulator not to offer a screening contract. A similar result is obtained by Kuhn \& Siciliani (2009) and Mougeot \& Naegelen (2014) in different settings. The underlying cause of non-responsiveness is the common value problem where the regulator's welfare is directly affected by the agent's type and not only by the transfer variable: ability in Kunh and Siciliani's model, severity in Mougeot \& Naegelen (2014) and altruism in our model. 


\section{Model}

A regulator contracts with an agent to jointly produce an outcome $S(a, e)$ such as health or education. We consider that the regulator and the agent contribute to this outcome with costly input $a$ and effort $e$, respectively. The regulator's input can take the form of hospitals or computing facilities, for example, while the agent's effort is a professional effort. The agent always observes the regulator's input $a$ while the regulator does not observe the agent's effort $e$. The setting so far is very similar to the one in Jelovac and Macho-Stadler (2002).

Both the regulator and the agent care for the outcome $S(a, e)$ but not to the same extend. The agent weights her utility from the outcome $S(a, e)$ by a non-negative parameter $\theta \leq 1$. An agent with a $\theta$ close to 1 is more altruistic or more mission-oriented than an agent with a low $\theta$.

We reasonably assume that the outcome function $S(a, e)$ is increasing in both input and effort and it is concave: $S_{a}>0, S_{e}>0, S_{a a}<0, S_{e e}<0$ and $S_{a a} S_{e e}-\left(S_{a e}\right)^{2}>0$. We also assume $S_{a e} \geq 0$. That is, we allow input and effort to be either complementary $\left(S_{a e}>0\right)$ or substitutes $\left(S_{a e}=0\right)$ in the production of the outcome $S$. To derive some of our results, it is useful to approximate the ratio $S_{a e} / S_{e e}$ by a negative constant: $S_{a e} / S_{e e}=-k$, with $k>0$. The unit cost of input $a$ and effort $e$ are $c$ and $v$, respectively. We denote by $\lambda$ the shadow cost of public funds $(\lambda>0)$.

The payment from the regulator to the agent is a simple transfer $t$. We rule out more sophisticated incentive payments because we consider that outcome and agent's effort cannot be accurately measured. Accordingly, we define the agent's utility and the regulator's welfare as follows:

$$
\begin{aligned}
& U=\theta S(a, e)+t-v e \\
& W=S(a, e)-(1+\lambda)(t+c a)+t-v e .
\end{aligned}
$$

Equation (1) is the agent's utility function. It comprises her transfer $t$ net of personal cost of effort ve, plus her direct benefit from the joint outcome $\theta S(a, e)$. Equation (2) is the regulator's welfare function. It is written as the sum of the surplus $S(a, e)$ and the provider's financial surplus net of regulator's direct costs. Note that to avoid double counting, the regulator does not take into the welfare function the direct benefit from the outcome derived by the agent. 


\section{First-best contract}

We derive in this section the optimal contract if the regulator can observe and contract upon the agent's effort $e$. It serves as a benchmark for our analysis.

The regulator chooses both the agent's effort $e$ and his own input $a$, as well as the level of compensation $t$ for the agent, so as to maximize welfare. He must ensure that the agent is willing and able to participate. We consider that the agent is willing to sign the contract only if her resulting utility is at least her reservation utility, normalized to zero for simplicity: $\theta S(a, e)+t-v e \geq 0$. However, the agent is able to participate only if her resulting budget allows her to: $t-v e \geq 0$. In our model, the agent's budget constraint always implies her participation constraint. Accordingly, our first-best problem is the following one:

$$
\max _{a, e, t} W=S(a, e)-(1+\lambda)(t+c a)+t-v e
$$

s.t.

$$
t-v e \geq 0
$$

which is equivalent to:

$$
\max _{a, e} W=S(a, e)-(1+\lambda)(v e+c a)
$$

with the budget constraint (BC) binding: $t=v e$.

The first-best input $a^{F B}$ and effort $e^{F B}$ are given by the first-order conditions for an interior solution and we summarise the first-best solution in the following lemma.

\section{Lemma 1. First-best solution analysis}

1. The first-best solution $\left(a^{F B}, e^{F B}, t^{F B}\right)$ is given by:

- $S_{a}\left(a^{F B}, e^{F B}\right)-(1+\lambda) c=0$

- $S_{e}\left(a^{F B}, e^{F B}\right)-(1+\lambda) v=0$

- $t^{F B}=v e^{F B}$

2. $a^{F B}, e^{F B}$ and $t^{F B}$ vary with the shadow cost of public funds according to:

- $\frac{\partial a^{F B}}{\partial \lambda}=\frac{c S_{e e}-v S_{e a}}{S_{a a} S_{e e}-\left(S_{a e}\right)^{2}}<0$

- $\frac{\partial e^{F B}}{\partial \lambda}=\frac{v S_{a a}-c S_{e a}}{S_{a a} S_{e e}-\left(S_{a e}\right)^{2}}<0$

- $\frac{\partial t^{F B}}{\partial \lambda}=v \frac{\partial e^{F B}}{\partial \lambda}<0$ 
3. $a^{F B}, e^{F B}$ and $t^{F B}$ are invariant to changes in altruism $\theta$.

The conditions defining the first-best input and effort reflect the traditional equality between marginal utility and marginal cost. The first-best transfer in Lemma 1 is given by the agent's budget constraint. This constraint binds so that the first-best transfer exactly compensates the disutility of first-best effort. The agent earns no rent. Lemma 1 also states that, first-best input, effort and transfer decrease with the shadow cost of public funds. This is intuitive since a higher burden on public expenses increases the marginal cost of effort and input. The variation in transfer just compensates for the variation in effort due to a change in the shadow cost of public funds. The first-best solution is invariant to the level of altruism of the agent because the altruism parameter $\theta$ appears neither in the budget constraint nor in the welfare function. Then, if the problem was only adverse selection (unknown altruism) without moral hazard (non-contractible effort), the first-best contract would be optimal. This is in line with the result of Choné \& Ma (2011) that, without heterogeneity on consumer's benefit, the regulator can impose the first-best quantity even under unknown altruism.

\section{Second-best contract}

The first-best solution does not account for situations in which, the agent's decision is not a contractible variable. We consider in the present section optimal contracts in a second-best regime where the regulator cannot contract upon the agent's endogenous effort. This is a case of moral hazard and the regulator anticipates that the agent can behave strategically if she is proposed the contract.

This situation is similar to a game in which the agent moves after the regulator and determines her optimal level of effort in stage 2 and the regulator takes into account this additional incentive constraint when he decides the transfer and his own level of input in stage 1.

The agent's problem is to choose the level of effort which maximizes her utility function (1). The first-order condition for the maximisation of the agent's utility implicitly defines the best-reply effort of the agent, $e=e^{*}(a, \theta)$, and it is given by:

$$
e=e^{*}(a, \theta) \Longleftrightarrow \theta S_{e}\left(e^{*}, a\right)-v=0
$$

The agent expands effort so that the marginal cost, $v$, equates the marginal utility, $\theta S_{e}\left(e^{*}, a\right)$, she derives from it. We notice at this stage that this effort increases with input $a$ as long as input and effort are complementary in the production of the outcome $S(a, e)$, that is, $S_{a e}>0$. It is also increasing in altruism. These relationships are all very 
intuitive and formally, they are given by:

$$
\begin{aligned}
& \frac{\partial e^{*}}{\partial a}=-\frac{S_{a e}}{S_{e e}}=k \geq 0 \\
& \frac{\partial e^{*}}{\partial \theta}=-\frac{S_{e}}{\theta S_{e e}}=-\frac{v}{\theta^{2} S_{e e}}>0 .
\end{aligned}
$$

The regulator's problem at stage 1 is to maximize the welfare function subject to the agent's budget constraint (BC) and the incentive compatibility constraint (ICC). The problem of the regulator is given by:

$$
\max _{a, t} W=S(a, e)-(1+\lambda)(t+c a)+t-v e
$$

s.t

$$
\begin{aligned}
& t-v e \geq 0 \\
& e=e^{*}(a, \theta) .
\end{aligned}
$$

Since the incentive constraint (ICC) does not depend on transfer $t$, the budget constraint (BC) binds as in the first-best analysis. Therefore, the problem is equivalent to:

$$
\max _{a} W=S\left(a, e^{*}(a, \theta)\right)-(1+\lambda)\left(c a+v e^{*}(a, \theta)\right) .
$$

We summarize in the following lemma the analysis of the second-best solution.

\section{Lemma 2. Second-best solution analysis}

1. The second-best solution $\left(a^{S B}, e^{S B}, t^{S B}\right)$ is given by:

- $S_{a}\left(a^{S B}, e^{S B}\right)-(1+\lambda) c-k v\left(1+\lambda-\frac{1}{\theta}\right)=0$

- $S_{e}\left(a^{S B}, e^{S B}\right)-\frac{1}{\theta} v=0$

- $t^{S B}=v e^{S B}$

2. $a^{S B}, e^{S B}$ and $t^{S B}$ vary with the shadow cost of public funds according to:

- $\frac{\partial a^{S B}}{\partial \lambda}=-\frac{1}{S_{a e}} \frac{v\left(S_{a e}\right)^{2}-c S_{a e} S_{e e}}{S_{a a} S_{e e}-\left(S_{a e}\right)^{2}}<0$

- $\frac{\partial e^{S B}}{\partial \lambda}=\frac{1}{S_{e e}} \frac{v\left(S_{a e}\right)^{2}-c S_{a e} S_{e e}}{S_{a a} S_{e e}-\left(S_{a e}\right)^{2}}<0$

- $\frac{\partial t^{S B}}{\partial \lambda}=v \frac{\partial e^{S B}}{\partial \lambda}<0$

3. $a^{S B}, e^{S B}$ and $t^{S B}$ vary with altruism according to:

- $\frac{\partial a^{S B}}{\partial \theta}=0$ 
- $\frac{\partial e^{S B}}{\partial \theta}=-\frac{v}{\theta^{2} S_{e e}}>0$

- $\frac{\partial t^{S B}}{\partial \theta}=-\frac{v^{2}}{\theta^{2} S_{e e}}>0$

The second-best effort increases with altruism since altruism increases the marginal benefit of effort to the agent without affecting the marginal cost of it. The secondbest transfer also increases with altruism to compensate the agent for her higher effort. Interestingly, input is invariant to changes in altruism. Altruism has no direct influence on either costs or benefits of the input.

It is interesting to see how agent's incentives change the optimal payment, effort and input. For this purpose, we compare the first-best and the second-best solutions. We summarise the comparison in the following lemma:

\section{Lemma 3. Comparison of the first-best and the second-best solutions}

- $a^{S B}=a^{F B}$

- $\left(e^{S B}, t^{S B}\right) \leq\left(e^{F B}, t^{F B}\right)$ if and only if $(1+\lambda) \theta \leq 1$

- $\left(e^{S B}, t^{S B}\right)>\left(e^{F B}, t^{F B}\right)$ if and only if $(1+\lambda) \theta>1$

The comparison presented in Lemma 3 shows to what extend moral hazard can be an issue for the regulator. When altruism is high (low), as well as the shadow cost of public funds, the level of effort chosen by the agent is distorted upward (downward) compared to the first-best contract. In fact, effort is increasing in altruism when decided by the agent (Lemma 2) while it is decreasing in the shadow cost of public funds when decided by the regulator (Lemma 1). This arises because the agent's marginal benefit from effort increases with altruism while the marginal regulator's cost of effort increases with the shadow cost of public funds when the regulator decides effort.

Note that when $(1+\lambda) \theta=1$, the agent is what we call a "perfect agent". She intervenes in the production of $S$ exactly as the regulator would have done if there were no delegation. A higher concern for patients would make her exert a higher effort than what would have been contracted upon, if effort was a contractible variable, because she does not internalize the shadow cost of public funds.

In all cases, the regulator's input is the same no matter whether the agent's effort is contractible or not.

Lemma 2 also states that, a more benevolent agent is given a higher transfer to compensate for a higher effort:

$$
\frac{\partial t^{S B}}{\partial \theta}>0
$$

If the regulator proposes the second-best contract to an heterogeneous population of agents and is able to tell agents apart according to their altruism, then very altruistic 
agents would work more and they would earn higher transfers than less altruistic agents. The regulator's input is the same for both types. If the regulator cannot distinguish between agents according to their altruism, less altruistic agents would pretend to be highly altruistic to earn a higher transfer. Therefore, the best the regulator can do is to propose different contracts depending on the type of the agent (whether she is very altruistic or not). We study in the next section optimal contracts in a third-best regime where we have moral hazard and heterogeneity among agents according to their concerns for the outcome.

\section{Third-best contracts}

We now consider that the regulator contracts with a population of agents who differ in their level of altruism and that he cannot tell the agents apart according to their altruism. To keep the analysis simple and interesting, we consider two different types of agents: altruistic agents who are more altruistic than a regulator's "perfect agent" $\left(\theta=\theta_{1}>\frac{1}{1+\lambda}\right)$ and selfish agents who are less altruistic than a "perfect agent" $\left(\theta=\theta_{0}<\frac{1}{1+\lambda}\right)$. We also refer to type 1 and type 0 to distinguish between them. We provide in Appendix $7.8 \mathrm{a}$ discussion on the consequence of this assumption on our results.

In this section, we consider that screening is possible and we assume that the regulator chooses a level of input together with a level of transfer for each type of agent: $\left(a_{1}, t_{1}\right)$ for the altruistic type of agent and $\left(a_{0}, t_{0}\right)$ for the selfish type of agent. As is typical in adverse selection problems, the regulator tailors contracts so that each type of agent exactly selects the one made for her. We assume that the regulator knows by experience that there is a proportion $\alpha$ of type 1 and $1-\alpha$ of type 0 in the population of agents. The regulator also anticipates moral hazard. That is, the regulator expects that agent's effort is $e=e^{*}(a, \theta)$, as defined by (ICC). The regulator solves the following problem: 


$$
\begin{aligned}
\max _{a_{0}, a_{1}, t_{0}, t_{1}} W= & \alpha\left\{S\left(a_{1}, e^{*}\left(a_{1}, \theta_{1}\right)\right)-(1+\lambda)\left(t_{1}+c a_{1}\right)+t_{1}-v e^{*}\left(a_{1}, \theta_{1}\right)\right\}+ \\
& (1-\alpha)\left\{S\left(a_{0}, e^{*}\left(a_{0}, \theta_{0}\right)\right)-(1+\lambda)\left(t_{0}+c a_{0}\right)+t_{0}-v e^{*}\left(a_{0}, \theta_{0}\right)\right. \\
\text { s.t } \quad & t_{0}-v e^{*}\left(a_{0}, \theta_{0}\right) \geq 0 \\
& t_{1}-v e^{*}\left(a_{1}, \theta_{1}\right) \geq 0 \\
& \theta_{0} S\left(a_{0}, e^{*}\left(a_{0}, \theta_{0}\right)\right)+t_{0}-v e^{*}\left(a_{0}, \theta_{0}\right) \geq \\
& \theta_{0} S\left(a_{1}, e^{*}\left(a_{1}, \theta_{0}\right)\right)+t_{1}-v e^{*}\left(a_{1}, \theta_{0}\right) ; \\
& \theta_{1} S\left(a_{1}, e^{*}\left(a_{1}, \theta_{1}\right)\right)+t_{1}-v e^{*}\left(a_{1}, \theta_{1}\right) \geq \\
& \theta_{1} S\left(a_{0}, e^{*}\left(a_{0}, \theta_{1}\right)\right)+t_{0}-v e^{*}\left(a_{0}, \theta_{1}\right) .
\end{aligned}
$$$$
(1-\alpha)\left\{S\left(a_{0}, e^{*}\left(a_{0}, \theta_{0}\right)\right)-(1+\lambda)\left(t_{0}+c a_{0}\right)+t_{0}-v e^{*}\left(a_{0}, \theta_{0}\right)\right\}
$$

The expected welfare is written as the weighted sum of the total surplus. The two first constraints, ( $\mathrm{BC} 0)$ and $(\mathrm{BC} 1)$, are budget constraints for type 0 and type 1 , respectively. As already mentioned in the previous sections, they ensure that each agent agrees to sign the contract. The two following constraints, (ICC0) and (ICC1), are adverse selection incentive compatibility constraints for type 0 and type 1 , respectively. They are set to ensure that each agent prefers the contract designed for her type. For $i \in\{0,1\}$, type $i$ agent must have a higher utility when she selects the contract that comprises transfer $t_{i}$ and regulator's input $a_{i}$, as compared to when she chooses the other contract made for type $j \neq i$.

The following lemma presents preliminary results, which are useful to simplify and to understand the regulator's problem.

\section{Lemma 4.}

1. The third-best solution $\left(e_{0}^{T B}, a_{0}^{T B}, t_{0}^{T B}, e_{1}^{T B}, a_{1}^{T B}, t_{1}^{T B}\right)$ must satisfy the following conditions:

$$
\begin{aligned}
& a_{1}^{T B} \geq a_{0}^{T B} \\
& t_{1}^{T B} \leq t_{0}^{T B} \\
& e_{1}^{T B}=e^{*}\left(a_{1}^{T B}, \theta_{1}\right)>e_{0}^{T B}=e^{*}\left(a_{0}^{T B}, \theta_{0}\right) .
\end{aligned}
$$

2. Constraints $(\mathrm{BC} 1)$ and $(\mathrm{ICC} 0)$ bind while $(\mathrm{BC} 0)$ does not.

3. Constraint (ICC1) holds as long as $a_{1}^{T B} \geq a_{0}^{T B}$. 
The main difference so far with the second-best analysis is that $(\mathrm{BC} 0)$ does not bind anymore and the transfer is not lower for a selfish agent. The reason relates to her incentive to pretend to be type 1 if the regulator proposed contracts designed as in the second-best analysis. This is why the third-best contract for a selfish agent must become more interesting to her. This justifies a higher transfer despite a lower level of altruism. This third-best transfer for a selfish agent not only compensates for her cost of effort but it also includes an informational rent.

Formally, (ICC0) binds and $(\mathrm{BC} 0)$ does not. From the binding constraints (ICC0) and $(\mathrm{BC} 1)$, we directly deduce the informational rent $R_{0}$ that is necessary for the type 0 agent not to pretend to be type 1:

$$
\begin{aligned}
R_{0}\left(a_{0}, a_{1}\right) & =t_{0}-v e^{*}\left(a_{0}, \theta_{0}\right) \\
& =v\left[e^{*}\left(a_{1}, \theta_{1}\right)-e^{*}\left(a_{1}, \theta_{0}\right)\right]+\theta_{0}\left[S\left(a_{1}, e^{*}\left(a_{1}, \theta_{0}\right)\right)-S\left(a_{0}, e^{*}\left(a_{0}, \theta_{0}\right)\right)\right] .
\end{aligned}
$$

This informational rent corresponds to the net financial surplus of the type 0 agent. The comparative statics for this informational rent directly follow:

$$
\begin{aligned}
& \frac{\partial R_{0}}{\partial a_{0}}=-\left[\theta_{0} S_{a}\left(a_{0}, e^{*}\left(a_{0}, \theta_{0}\right)\right)+k v\right]<0 ; \\
& \frac{\partial R_{0}}{\partial a_{1}}=\theta_{0} S_{a}\left(a_{1}, e^{*}\left(a_{1}, \theta_{0}\right)\right)+k v>0 .
\end{aligned}
$$

Thus, the informational rent of a type- 0 agent decreases with $a_{0}$ and it increases with $a_{1}$. This is due to the direct effect of the regulator's input $a$ on the agent's personal satisfaction from the outcome $\theta S(a, e)$, even if this direct effect is not very strong given the low level of altruism of a selfish agent. A higher level of input for the selfish type increases her satisfaction and accordingly reduces the informational rent that is necessary for her to prefer her contract over the one of an altruistic agent. Conversely, if the input increases for the altruistic agent, her contract becomes all the more interesting for the selfish agent and a higher informational rent must prevent the selfish agent from taking the contract designed for the type-1 agent.

We use the results of Lemma 4 to simplify the regulator's problem as follows, with the best-reply effort, $e=e^{*}(a, \theta)$, implicitly defined in the incentive compatibility constraint 
(ICC):

$$
\begin{aligned}
\max _{a_{0}, a_{1}} W= & \alpha\left\{S\left(a_{1}, e^{*}\left(a_{1}, \theta_{1}\right)\right)-(1+\lambda)\left(c a_{1}+v e^{*}\left(a_{1}, \theta_{1}\right)\right)\right\} \\
& +(1-\alpha)\left\{S\left(a_{0}, e^{*}\left(a_{0}, \theta_{0}\right)\right)-(1+\lambda)\left(c a_{0}+v e^{*}\left(a_{0}, \theta_{0}\right)\right)\right\} \\
& -(1-\alpha) \lambda R_{0}\left(a_{0}, a_{1}\right) \\
\text { s.t } & \\
& a_{1} \geq a_{0} .
\end{aligned}
$$

The important difference with the former sections is that welfare now accounts for the informational rent (in the last term of $W$ ) that is necessary to prevent type 0 agents from pretending to be type 1 . The following lemma presents the third-best solution.

\section{Lemma 5. Third-best solution}

The third-best solution $\left(a_{0}^{T B}, e_{0}^{T B}, t_{0}^{T B}, a_{1}^{T B}, e_{1}^{T B}, t_{1}^{T B}\right)$ is given by:

- $a_{0}^{T B}=a_{1}^{T B}=a^{T B}=a^{S B}$

- $S_{e}\left(a^{T B}, e_{0}^{T B}\right)-\frac{1}{\theta_{0}} v=0$

- $S_{e}\left(a^{T B}, e_{1}^{T B}\right)-\frac{1}{\theta_{1}} v=0$

- $t_{0}^{T B}=t_{1}^{T B}=v e_{1}^{T B}$

Lemma 5 tells us that no screening contract dominates the second-best contract designed in Section 4. The best thing a regulator can do is to design a contract as in the second-best analysis, expecting every agent to sign up the contract designed for the altruistic type. The difference in altruism between agents does not justify different regulator's inputs per se. The only effect of screening according to input would be to increase the informational rent of the selfish agents. This conflict between optimality and efficiency is referred to as "non-responsiveness" (See Guenesrie and Laffont, 1986). Giving a higher input to altruistic agents will satisfy the monotonicity condition but it will be too costly for the regulator because of the higher rent it implies for the selfish agents.

\section{Proposition 1. Comparison between first-, second- and third-best solutions}

- Ranking of regulator's input levels: $a^{F B}=a_{0}^{S B}=a_{1}^{S B}=a_{0}^{T B}=a_{1}^{T B}$

- Ranking of agents' effort levels: $e_{0}^{S B}=e_{0}^{T B}<e^{F B}<e_{1}^{S B}=e_{1}^{T B}$

- Ranking of transfer levels: $t_{0}^{S B}<t^{F B}<t_{1}^{S B}=t_{0}^{T B}=t_{1}^{T B}$ 
Adding adverse selection to our problem does not change the optimal contracts. We already mentioned in Section 3 that the first-best contract continues to be optimal if there were adverse selection without moral hazard. Indeed, a first-best contract in this setting does not vary with altruism anyway. When we consider in this section moral hazard together with adverse selection, we show that there is no screening contract that dominates a pooling contract designed to deal with moral hazard for an altruistic agent. Indeed, a screening contract cannot optimally separate types without increasing the costs of the informational rent obtained by the selfish agents.

The level of the regulator's input is identical across all regimes. Differentiating inputs according to altruism would only increase the informational rent of the selfish agents without getting closer to an efficient solution. Levels of effort are therefore identical across second-best and third-best regimes. Altruistic (selfish) agents exert a higher (lower) effort than a "perfect agent". Accordingly, the transfer under the second-best regime is higher (lower) than the one of a "perfect agent" or, equivalently, the one in the first-best regime. The pooling contract in the third-best regime imposes a unique level of transfer that coincides with the second-best transfer for the altruistic agents.

\section{Concluding remarks}

In this paper, we analyze a particular class of regulator-agent relationships. The main features of our model are joint participation to the non-contractible outcome, different non-contractible concern for the outcome being produced and non-contractible effort. Transfer and regulator's input are the two instruments to solve information asymmetry. This setting is relevant for sectors of activities such as education and health.

Comparing first- and second-best solutions to analyze the effect of moral hazard, we show that the provider's input is not distorted by moral hazard while the agent's effort as well as the corresponding transfer are distorted upward (downward) when altruism and the shadow cost of public funds are high (low). In our analysis of the consequences of combined adverse selection and moral hazard, we show that, a separating contract is dominated by a pooling contract; regulator's input and agent's effort are identical to the second-best ones; and the transfer is unique and high enough to compensate an altruistic agent for her effort.

Our main result suggests that screening between altruistic and selfish agents is not optimal in our setting. This result may explain why sectors such as health and education hardly rely on screening. Extracting information about altruism is indeed too costly for the regulator. In this situation, the regulator can only expect that selfish agents sign the contract that deals with moral hazard of altruistic agents. 


\section{Appendices}

\subsection{Proof of Lemma 1}

Proof. The first-best solution under point 1 directly follows the maximization of the regulator's welfare (P1'). The comparative statics under points 2 and 3 result from the total differentiation of the second-best solution under point 1 .

\subsection{Proof of Lemma 2}

Proof. The second-best solution under point 1 directly follows the maximization of the regulator's welfare (P2'). The comparative statics under points 2 and 3 result from the total differentiation of the second-best solution under point 1 .

\subsection{Proof of Lemma 3}

Proof. When $(1+\lambda) \theta=1$, the second-best solution coincides with the first-best solution. Moreover, the third result in Lemma 2 states that $a^{S B}$ is invariant to changes in altruism $\theta$ while $e^{S B}$ and $t^{S B}$ both increase with $\theta$. Using this result together with the approximation $S_{a e} / S_{e e}=-k$, directly yields the result in Lemma 3 .

\subsection{Proof of Lemma 4}

\section{Proof. 1. Proof of $a_{1}^{T B} \geq a_{0}^{T B}$}

We define the function $F(a, \theta)=\theta S\left(a, e^{*}(a, \theta)\right)-v e^{*}(a, \theta)$, with $e^{*}(a, \theta)$ implicitly defined by $\theta S_{e}^{*}(a, e)-v=0$, as in the initial incentive compatibility constraint (ICC). Our modeling assumptions imply $F_{a}(a, \theta)=\theta S_{a}>0 ; F_{\theta}(a, \theta)=S>0$; $F_{\theta a}(a, \theta)=S_{a}+k S_{e}>0$.

We sum the constraints (ICC0) and (ICC1) and rearrange terms to write this sum as $F\left(a_{1}, \theta_{1}\right)-F\left(a_{1}, \theta_{0}\right) \geq F\left(a_{0}, \theta_{1}\right)-F\left(a_{0}, \theta_{0}\right)$.

We define $G(a)=F\left(a, \theta_{1}\right)-F\left(a, \theta_{0}\right)$ and we rewrite the sum of (ICC0) and (ICC1) as $G\left(a_{1}\right) \geq G\left(a_{0}\right)$. This inequality holds iff $a_{1}^{T B} \geq a_{0}^{T B}$ since $G^{\prime}(a)=F_{a}\left(a, \theta_{1}\right)$ $F_{a}\left(a, \theta_{0}\right)>0$ for $\theta_{1}>\theta_{0}$.

2. Proof of $e_{1}^{T B}>e_{0}^{T B}$

The best-reply effort is implicitly defined by $\theta S_{e}\left(a, e^{*}(a, \theta)\right)-v=0$, as in (ICC). Equations (3) and (4) establish that the best-reply effort increases in input $a$ and in altruism $\theta$. Therefore, $a_{1}^{T B} \geq a_{0}^{T B}$ and $\theta_{1}>\theta_{0}$ directly imply $e_{1}^{T B}>e_{0}^{T B}$. 
3. Proof of $t_{1}^{T B} \leq t_{0}^{T B}$

We rewrite (ICC0) as $F\left(a_{0}, \theta_{0}\right)+t_{0} \geq F\left(a_{1}, \theta_{0}\right)+t_{1}$. This inequality implies $t_{1}^{T B} \leq$ $t_{0}^{T B}$ since $F_{a}(a, \theta)>0$ and $a_{1}^{T B} \geq a_{0}^{T B}$.

4. Proof of $t_{0}^{T B}-v e_{0}^{T B}>0$ (i.e., $(\mathrm{BC} 0)$ does not bind)

We have just proved that $t_{1}^{T B} \leq t_{0}^{T B}$. Moreover, $t_{1}^{T B} \geq v e_{1}^{T B}$ by $(\mathrm{BC} 1)$ and $v e_{1}^{T B}>$ $v e_{0}^{T B}$ because $e_{1}^{T B}>e_{0}^{T B}$. Therefore, $t_{0}^{T B} \geq t_{1}^{T B} \geq v e_{1}^{T B}>v e_{0}^{T B}$ and therefore, $t_{0}^{T B}>v e_{0}^{T B}$.

5. Proof that $(\mathrm{ICC} 0)$ is binding

Regulator's welfare $W$ is decreasing in $t_{0}$ and $(\mathrm{BC} 0)$ does not bind. Therefore, $t_{0}^{T B}$ is the lowest one that satisfies all constraints and thus (ICC0) binds.

6. Proof that (ICC1) holds as long as $a_{1} \geq a_{0}$

We substitute the binding (ICC0) into (ICC1) and we rewrite (ICC1) as $G\left(a_{1}\right) \geq$ $G\left(a_{0}\right)$, which holds iff $a_{1} \geq a_{0}$.

7. Proof that $(\mathrm{BC} 1)$ is binding

Regulator's welfare $W$ is decreasing in $t_{1}$ and (ICC1) holds as long as $a_{1} \geq a_{0}$. Therefore, $t_{1}^{T B}$ is the lowest one that satisfies all constraints and thus (BC1) binds.

\subsection{Proof of Lemma 5}

Proof. Denoting $\mu$ the Lagrange multiplier associated to the constraint $a_{1} \geq a_{0}$, we write the first-order conditions of the simplified third-best maximization problem (P3') as follows:

$$
\begin{aligned}
& \frac{\partial W}{\partial a_{0}}=(1-\alpha)\left[\left(1+\lambda \theta_{0}\right)\left(S_{a}\left(a_{0}, e^{*}\left(a_{0}, \theta_{0}\right)\right)+\frac{k v}{\theta_{0}}\right)-(1+\lambda)(c+k v)\right]-\mu=0 \\
& \frac{\partial W}{\partial a_{1}}=\alpha\left[S_{a}\left(a_{1}, e^{*}\left(a_{1}, \theta_{1}\right)\right)+\frac{k v}{\theta_{1}}-(1+\lambda)(c+k v)\right] \\
& -(1-\alpha) \lambda \theta_{0}\left[S_{a}\left(a_{1}, e^{*}\left(a_{1}, \theta_{0}\right)\right)+\frac{k v}{\theta_{0}}\right]+\mu=0 .
\end{aligned}
$$


We define $f(a, \theta)=S_{a}\left(a, e^{*}(a, \theta)\right)+\frac{k v}{\theta}$ and we rewrite these first-order conditions as:

$$
\begin{aligned}
& \frac{\partial W}{\partial a_{0}}=(1-\alpha)\left[\left(1+\lambda \theta_{0}\right) f\left(a_{0}, \theta_{0}\right)-(1+\lambda)(c+k v)\right]-\mu=0 \\
& \frac{\partial W}{\partial a_{1}}=\alpha\left[f\left(a_{1}, \theta_{1}\right)-(1+\lambda)(c+k v)\right]-(1-\alpha) \lambda \theta_{0} f\left(a_{1}, \theta_{0}\right)+\mu=0 .
\end{aligned}
$$

Using Equations (3) and (4), we notice that

$$
f_{a}(a, \theta)=\frac{S_{a a} S_{e e}-S_{a e}^{2}}{S_{e e}}<0
$$

and

$$
f_{\theta}(a, \theta)=S_{a e} \frac{\partial e^{*}}{\partial \theta}-\frac{k v}{\theta^{2}}=0 .
$$

The fact that $f_{\theta}(a, \theta)=0$ allows us to rewrite the first-order conditions as:

$$
\begin{aligned}
& \frac{\partial W}{\partial a_{0}}=(1-\alpha)\left[\left(1+\lambda \theta_{0}\right) f\left(a_{0}\right)-(1+\lambda)(c+k v)\right]-\mu=0 ; \\
& \frac{\partial W}{\partial a_{1}}=\left[\alpha-(1-\alpha) \lambda \theta_{0}\right] f\left(a_{1}\right)-\alpha(1+\lambda)(c+k v)+\mu=0 ;
\end{aligned}
$$

where $f(a)=f\left(a, \theta_{0}\right)=f\left(a, \theta_{1}\right)$ and where $f^{\prime}(a)=f_{a}(a, \theta)<0$. We impose $\alpha-(1-$ $\alpha) \lambda \theta_{0}>0$ to have a well-defined maximization problem (otherwise, (P3') would not be concave in $a_{1}$ and the solution would be $a_{0}=a_{1}=0$ ). If $\mu=0$, the first-order conditions imply

$$
f\left(a_{0}\right)=\frac{1+\lambda}{1+\lambda \theta_{0}}(c+k v)<f\left(a_{1}\right)=\frac{\alpha(1+\lambda)}{\alpha-(1-\alpha) \lambda \theta_{0}}(c+k v)
$$

which itself implies $a_{0}>a_{1}$. This contradicts the constraint $a_{1} \geq a_{0}$. Therefore, $\mu \neq 0$ and the constraint binds: $a_{1}=a_{0}$.

We then solve the maximization program with a unique input level $a$ and we find the following first-order condition:

$$
\begin{aligned}
& \frac{\partial W}{\partial a}=\alpha\left(S_{a}\left(a, e^{*}\left(a, \theta_{1}\right)\right)-(1+\lambda) c-k v\left(1+\lambda-\frac{1}{\theta_{1}}\right)\right) \\
& +(1-\alpha)\left(S_{a}\left(a, e^{*}\left(a, \theta_{0}\right)\right)-(1+\lambda) c-k v\left(1+\lambda-\frac{1}{\theta_{0}}\right)\right)=0 .
\end{aligned}
$$

This first-order condition is a convex combination of first-order conditions defining the 
second-best input $a^{S B}$ for altruism levels $\theta_{1}$ and $\theta_{0}$, respectively (see Lemma 2, point 1). Since $a^{S B}$ is invariant to changes in altruism (Lemma 2, point 3), then a convex combination of $a^{S B}$ for different levels of altruism is also invariant to changes in altruism. Therefore,

$$
a_{0}^{T B}=a_{1}^{T B}=a^{T B}=\alpha a_{1}^{S B}+(1-\alpha) a_{0}^{S B}=a^{S B} .
$$

Effort levels are implicitly defined by the initial incentive compatibility constraint (ICC0):

$$
\theta_{i} S_{e}\left(a^{T B}, e_{i}^{T B}\right)-v=0, i=0,1
$$

Given $a_{0}^{T B}=a_{1}^{T B}$, we need $t_{0}^{T B}=t_{1}^{T B}$ for (ICC0) to bind, and we need $t_{1}^{T B}=v e_{1}^{T B}$ for (BC1) to bind. Therefore,

$$
t_{0}^{T B}=t_{1}^{T B}=v e_{1}^{T B}
$$

\subsection{Proof of Proposition 1}

Proof. Straightforward from the preceding lemmata.

\subsection{Illustrative example}

To illustrate our analysis, we provide an example with the following functional form for the joint outcome $S(a, e)$ :

$$
S(a, e)=a-\frac{a^{2}}{2}+e-\frac{e^{2}}{2}+k a e
$$

where $k \in[0,1)$ is a constant. This quadratic function satisfies all of our modelling assumptions.

\subsubsection{First-best analysis}

The first-best contract solves the following program:

$$
\max _{a, e, t} W=a-\frac{a^{2}}{2}+e-\frac{e^{2}}{2}+k a e-(1+\lambda)(t+c a)+t-v e
$$

s.t

$$
t-v e \geq 0
$$


The first-best solution (Lemma 1) is given by:

- $a^{F B}=\frac{(1+k)-(1+\lambda)(k v+c)}{1-k^{2}}$

- $e^{F B}=\frac{(1+k)-(1+\lambda)(k c+v)}{1-k^{2}}$

- $t^{F B}=v e^{F B}$

The comparative statics (Lemma 1) are as follow:

1. $a^{F B}, e^{F B}$ and $t^{F B}$ are invariant to changes in $\theta$.

2. $a^{F B}, e^{F B}$ and $t^{F B}$ vary with $\lambda$ according to:

- $\frac{\partial a^{F B}}{\partial \lambda}=-\frac{k v+c}{1-k^{2}}<0$

- $\frac{\partial e^{F B}}{\partial \lambda}=-\frac{k c+v}{1-k^{2}}<0$

- $\frac{\partial t^{F B}}{\partial \lambda}=v \frac{\partial e^{F B}}{\partial \lambda}<0$

\subsubsection{Second-best analysis}

The second-best contract solves the following program:

$$
\max _{a, e, t} W=a-\frac{a^{2}}{2}+e-\frac{e^{2}}{2}+k a e-(1+\lambda)(t+c a)+t-v e
$$

s.t

$$
\begin{aligned}
& t-v e \geq 0 \\
& e=e^{*}(a, \theta)=1+k a-\frac{v}{\theta}
\end{aligned}
$$

The second-best solution (Lemma 2) is given by:

- $a^{S B}=\frac{(1+k)-(1+\lambda)(k v+c)}{1-k^{2}}$

- $e^{S B}=k\left\{\frac{(1+k)-(1+\lambda)(c+v k)}{1-k^{2}}\right\}+1-\frac{v}{\theta}$

- $t^{S B}=v e^{S B}$

The comparative statics (Lemma 2) are as follow:

1. $a^{S B}, e^{S B}$ and $t^{S B}$ vary with $\lambda$ according to:

- $\frac{\partial a^{S B}}{\partial \lambda}=-\frac{c+v k}{1-k^{2}}<0$

- $\frac{\partial e^{S B}}{\partial \lambda}=-k \frac{(c+v k)}{1-k^{2}}<0$ 
- $\frac{\partial t^{S B}}{\partial \lambda}=v \frac{\partial e^{S B}}{\partial \lambda}<0$

2. $a^{S B}, e^{S B}$ and $t^{S B}$ vary with $\theta$ according to:

- $\frac{\partial a^{S B}}{\partial \theta}=0$

- $\frac{\partial e^{S B}}{\partial \theta}=\frac{v}{\theta^{2}}>0$

- $\frac{\partial t^{S B}}{\partial \theta}=v \frac{\partial e^{S B}}{\partial \theta}>0$

First- and second-best solutions compare as follow (Lemma 3):

- $a^{S B}=a^{F B}$

- $\left(e^{S B}, t^{S B}\right) \leq\left(e^{F B}, t^{F B}\right)$ if and only if $(1+\lambda) \theta \leq 1$

- $\left(e^{S B}, t^{S B}\right)>\left(e^{F B}, t^{F B}\right)$ if and only if $(1+\lambda) \theta>1$

\subsubsection{Third-best analysis}

The third-best contract solves the following program (after simplification using the results of Lemma 4):

$$
\begin{aligned}
\max _{a_{0}, a_{1}} W= & \alpha\left\{a_{1}-\frac{a_{1}^{2}}{2}+\frac{1}{2}\left(1+k a_{1}\right)^{2}-\frac{1}{2}\left(\frac{v}{\theta_{1}}\right)^{2}-(1+\lambda)\left[c a_{1}+v\left(1+k a_{1}-\frac{v}{\theta_{1}}\right)\right]\right\}+ \\
& (1-\alpha)\left\{a_{0}-\frac{a_{0}^{2}}{2}+\frac{1}{2}\left(1+k a_{0}\right)^{2}-\frac{1}{2}\left(\frac{v}{\theta_{0}}\right)^{2}-(1+\lambda)\left[c a_{0}+v\left(1+k a_{0}-\frac{v}{\theta_{0}}\right)\right]\right\} \\
& -(1-\alpha) \lambda R_{0}\left(a_{0}, a_{1}\right)
\end{aligned}
$$

s.t

$$
a_{1} \geq a_{0}
$$

where

$$
R_{0}\left(a_{0}, a_{1}\right)=v^{2}\left\{\frac{1}{\theta_{0}}-\frac{1}{\theta_{1}}\right\}+\theta_{0}(1+k)\left(a_{1}-a_{0}\right)\left\{1-\frac{(1-k)\left(a_{1}+a_{0}\right)}{2}\right\}
$$

Let us denote by $\mu$ the Lagrange multiplier associated with the constraint. The first-order conditions write as follows: 


$$
\begin{aligned}
& \frac{\partial W}{\partial a_{0}}=(1-\alpha)\left\{(1+k)\left(1+\lambda \theta_{0}\right)\left(1-(1-k) a_{0}\right)-(1+\lambda)(c+k v)\right\}-\mu=0 \\
& \frac{\partial W}{\partial a_{1}}=(1+k)\left(\alpha-(1-\alpha) \lambda \theta_{0}\right)\left(1-(1-k) a_{1}\right)-\alpha(1+\lambda)(c+k v)+\mu=0 .
\end{aligned}
$$

- For $\mu=0$, we have $a_{1}$ and $a_{0}$ given by:

$$
\begin{aligned}
& a_{0}^{T B}=\frac{1}{1-k}\left\{1-\frac{(1+\lambda)(c+k v)}{(1+k)\left(1+\lambda \theta_{0}\right)}\right\} \\
& a_{1}^{T B}=\frac{1}{1-k}\left\{1-\frac{\alpha(1+\lambda)(c+k v)}{(1+k)\left(\alpha-(1-\alpha) \lambda \theta_{0}\right)}\right\} .
\end{aligned}
$$

We thus have $a_{1}<a_{0}$, which contradicts the constraint $a_{1} \geq a_{0}$. Therefore, we cannot have $\mu=0$ and the constraint $a_{1} \geq a_{0}$ must bind.

- For $\mu \neq 0$, there is a single level of $a=a^{T B}$ which is the solution to the following program:

$$
\max _{a} W=a-\frac{a^{2}}{2}+\frac{1}{2}(1+k a)^{2}-(1+\lambda)[c a+v(1+k a)]+\left\{\lambda+\alpha-\frac{\alpha}{2 \theta_{1}}\right\} \frac{v^{2}}{\theta_{1}}+(1-\alpha)\left\{1-\frac{1}{2 \theta_{0}}\right\} \frac{v^{2}}{\theta_{0}} .
$$

The third-best solution (Lemma 5) is given by:

- $a^{T B}=\frac{(1+k)-(1+\lambda)(k v+c)}{1-k^{2}}$

- $e_{1}^{T B}=k\left\{\frac{(1+k)-(1+\lambda)(c+v k)}{1-k^{2}}\right\}+1-\frac{v}{\theta_{1}}$

- $e_{0}^{T B}=k\left\{\frac{(1+k)-(1+\lambda)(c+v k)}{1-k^{2}}\right\}+1-\frac{v}{\theta_{0}}$

- $t_{1}^{T B}=v e_{1}^{T B}$

- $t_{0}^{T B}=v e_{0}^{T B}$

The ranking of the first-, second- and third-best solution (Proposition 1) is straightforward.

\subsection{Rankings of altruism with respect to the shadow cost of public funds}

We assume in the third-best analysis that $\theta_{0}<\frac{1}{1+\lambda}<\theta_{1}$. This assumption only affects the comparison between first-, second- and third-best analyses (Proposition 1). The formal analyses in each sub-section do not depend on this assumption. 
If we assume instead, that no agent is more altruistic than the perfect agent, $\theta_{0}<$ $\theta_{1}<\frac{1}{1+\lambda}$, Proposition 1 would state the following:

- $a^{F B}=a_{0}^{S B}=a_{1}^{S B}=a_{0}^{T B}=a_{1}^{T B}$

- $e_{0}^{S B}=e_{0}^{T B}<e_{1}^{S B}=e_{1}^{T B}<e^{F B}$

- $\mathrm{t}_{0}^{S B}<t_{1}^{S B}=t_{0}^{T B}=t_{1}^{T B}<t^{F B}$

The only change with what we have derived in our main result is the fact that, effort and transfer of a perfect agent are always higher than those of both types of agent.

\section{References}

Barigozzi, Francesca, \& Burani, Nadia. 2016. Competition and screening with motivated health professionals. Journal of Health Economics, 50, 358-371.

Biglaiser, Gary, \& Ma, Albert Ching-to. 2007. Moonlighting: public service and private practice. The RAND Journal of Economics, 38(4), 1113-1133.

Brosig-Koch, Jeannette, Hennig-Schmidt, Heike, Kairies-Schwarz, Nadja, \& Wiesen, Daniel. 2017. The Effects of Introducing Mixed Payment Systems for Physicians: Experimental Evidence. Health Economics, 26(2), 243-262.

Chalkley, Martin, \& Malcomson, James M. 1998. Contracting for health services when patient demand does not reflect quality. Journal of health economics, 17(1), 1-19.

Choné, Philippe, \& Ma, Ching-to Albert. 2011. Optimal health care contract under physician agency. Annals of Economics and Statistics/Annales d'Économie et de Statistique, $229-256$.

Dor, Avi, \& Watson, Harry. 1995. The hospital-physician interaction in US hospitals: evolving payment schemes and their incentives. European Economic Review, 39(3), 795-802.

Guesnerie, Roger, \& Laffont, Jean-Jacques. 1984. A complete solution to a class of principal-agent problems with an application to the control of a self-managed firm. Journal of public Economics, 25(3), 329-369.

Hammond, Peter. 1987. Altruism. The New Palgrave: A Dictionary of Economics. Macmillan, London, 85-87.

Jack, William. 2005. Purchasing health care services from providers with unknown altruism. Journal of health economics, 24(1), 73-93. 
Jelovac, Izabela, \& Macho-Stadler, Inés. 2002. Comparing organizational structures in health services. Journal of Economic Behavior \& Organization, 49(4), 501-522.

Kairies-Schwarz, Nadja. 2014. Altruism Heterogeneity and Quality Competition Among Healthcare Providers. Ruhr Economic Papers.

Köszegi, Botond. 2014. Behavioral contract theory. Journal of Economic Literature, 52(4), 1075-1118.

Kuhn, Michael, \& Siciliani, Luigi. 2009. Performance indicators for quality with costly falsification. Journal of Economics \& Management Strategy, 18(4), 1137-1154.

Kwakman, Kitty. 2003. Factors affecting teachers' participation in professional learning activities. Teaching and teacher education, 19(2), 149-170.

Laffont, Jean-Jacques, \& Martimort, David. 2009. The theory of incentives: the principalagent model. Princeton university press.

Liu, Ting, \& Ma, Ching-to Albert. 2013. Health insurance, treatment plan, and delegation to altruistic physician. Journal of Economic Behavior \& Organization, 85, 79-96.

Macho-Stadler, Ines, \& Pérez Castrillo, David. 2016. Moral hazard: Base models and two extensions.

Monson, Verna, \& Hamilton, Neil W. 2010. Ethical professional (trans) formation: Early career lawyers make sense of professionalism.

Mougeot, Michel, \& Naegelen, Florence. 2014. Asymmetric Information and Pooling Contracts in the Hospital Sector. Journal of Institutional and Theoretical Economics JITE, 170(2), 365-386.

Mussa, Michael, \& Rosen, Sherwin. 1978. Monopoly and product quality. Journal of Economic theory, 18(2), 301-317.

Naegelen, Florence, \& Mougeot, Michel. 2011. Power of incentives with motivated agents in public organizations. Journal of Public Economic Theory, 13(3), 391-416.

Salant, Stephen W. 1989. When is inducing self-selection suboptimal for a monopolist? The Quarterly Journal of Economics, 104(2), 391-397.

Spence, A Michael. 1980. Multi-product quantity-dependent prices and profitability constraints. The Review of Economic Studies, 47(5), 821-841.

Spence, Michael. 1977. Nonlinear prices and welfare. Journal of public economics, 8(1), $1-18$. 
Stokey, Nancy L. 1979. Intertemporal price discrimination. The Quarterly Journal of Economics, 355-371.

Watt, Helen M.G., Richardson, Paul W., \& Wilkins, Kari. 2014. Profiles of professional engagement and career development aspirations among USA preservice teachers. International Journal of Educational Research, 65(Supplement C), 23 - 40. 